Running Title: Caerin 1 antimicrobial peptides inhibit HIV

\title{
Inhibition of HIV Infection by Caerin 1 Antimicrobial Peptides
}

\author{
Scott VanCompernolle ${ }^{1 *}$, Patricia B. Smith ${ }^{1}$, John H. Bowie ${ }^{2}$, \\ Michael J. Tyler ${ }^{3}$, Derya Unutmaz ${ }^{1,4}$, and Louise A. Rollins-Smith ${ }^{5 \dagger}$
}

Department of Pathology, Microbiology and Immunology Vanderbilt University School of Medicine, Nashville, TN 37232 ${ }^{1}$; Department of Chemistry, The University of Adelaide, Australia ${ }^{2}$; Department of Environmental Biology, The University of Adelaide, Australia ${ }^{3}$; Department of Microbiology, The Jackson Laboratory for Genomic Medicine, Farmington, CT, 06032 ${ }^{4}$; Departments of Pathology, Microbiology and Immunology, and Pediatrics, Vanderbilt University School of Medicine, Nashville, TN 37232 and Department of Biological Sciences, Vanderbilt University, Nashville, TN $37235^{5}$

${ }^{\dagger}$ Corresponding author. Mailing address: Department of Pathology, Microbiology and Immunology, A-5301 Medical Center North, Vanderbilt University School of Medicine, Nashville, TN 37273. Phone: (615-343-4119); Fax: (615) 343-7932; E-mail: louise.rollinssmith@vanderbilt.edu

${ }^{*}$ Current address, Shook, Hardy and Bacon, L.L.P., Kansas City, MO 64108

\begin{abstract}
The major mode of transmission of the human immunodeficiency virus (HIV) is by sexual intercourse. In the effort to halt the spread of HIV, one measure that holds great promise is the development of effective microbicides that can prevent transmission. Previously we showed that several amphibian antimicrobial peptides (AMPs) completely inhibit HIV infection of T cells while maintaining good viability of the $\mathrm{T}$ cell targets. These peptides also inhibited the transfer of HIV by dendritic cells (DCs) to T cells when added up to eight hours after virus exposure. Here we report on the anti-HIV activity of 18 additional structurally related caerin 1 family peptides in comparison with the our previous best candidate caerin 1.9. Nine peptides were equally effective or more effective in the inhibition of T cell infection and disruption of the HIV envelope as caerin 1.9. Of those nine peptides, three peptides (caerin 1.2, caerin 1.10, and caerin 1.20) exhibited excellent inhibition of HIV infectivity at low concentrations $(12-25 \mu \mathrm{M})$ and limited toxicity against target $\mathrm{T}$ cells and endocervical epithelial cells. There was a direct correlation between the effectiveness of the peptides in disruption of the viral envelope and their capacity to inhibit infection. Thus, several additional caerin 1 family peptides inhibit HIV infection, have limited toxicity for vaginal epithelial cells, and would be good candidates for inclusion in microbicide formulations.
\end{abstract}

Keywords: antimicrobial peptides, caerin 1, endocervical epithelial cells, human immunodeficiency virus, lymphocytes 


\section{Introduction}

The human immunodeficiency virus (HIV) continues to cause great human suffering worldwide as infected individuals develop acquired immunodeficiency syndrome (AIDS) and secondary infections resulting from impaired immunity. The major mode of transmission of HIV is by sexual intercourse [22]. Until an effective vaccine for this disease is developed, there is a compelling need for other strategies to prevent transmission. An antimicrobial gel or cream capable of inhibiting transmission of HIV at mucosal sites could significantly impact prevention efforts. Antimicrobial peptides (AMPs) from amphibian skin hold great potential to minimize or even prevent HIV transmission. Anuran amphibians (frogs and toads) provide a rich source of broad-spectrum AMPs that have been shown to have activity against a variety of microbes including Gram positive and Gram negative bacteria, fungi, and viruses [4, 13, 30]. We and others have previously demonstrated the capacity of amphibian AMPs to completely inhibit HIV infection of T cells by disruption of the virion membrane while maintaining target cell viability $[11,25$ reviewed in 8,29]. These amphibian peptides were also highly effective in the inhibition of the transfer of HIV by dendritic cells (DCs) to T cells [11,25], even when DCs were transiently exposed to peptides eight hours after virus capture [25]. We hypothesized that related peptides could be even more potent and less toxic. Here we report on the activity of 18 additional structurally related caerin 1 peptides (in addition to the previously tested caerin 1.1 and caerin 1.9) tested for their ability to inhibit HIV infection of T cells, their ability to disrupt the HIV envelope and release viral core protein (p24), and their toxicity to target T cells and vaginal epithelial cells lines. A number of additional AMPs were identified with excellent qualities of inhibition of HIV infection and limited toxicity to T cells and epithelial cells.

\section{Materials and methods}

Antimicrobial peptides

The antimicrobial peptide sequences, species of origin, and mass are listed in Table 1. All peptides were synthesized by Chiron Mimotopes (Clayton, Victoria, Australia) or Genscript Corporation (Piscataway, NJ, USA) using L-amino acids and standard 9fluorenylmethoxycarbonyl (Fmoc) chemistry.

\section{Primary cell isolation, cell lines and cell culture}

Peripheral blood mononuclear cells were isolated from adult blood using a FicollHypaque gradient. Monocytes and $\mathrm{CD}^{+} \mathrm{T}$ cells were isolated from the peripheral blood mononuclear cell population as previously described $[12,15,25]$. The human endocervical cell line End1/E6E7 (CRL-2615) was purchased from the ATCC. This cell line was derived from human cells immortalized with human papilloma virus 16/E6E7. The endocervical cell line was cultured in keratinocyte serum-free medium (GIBCO ${ }^{\circledR}$ Grand Island, NY) supplemented with bovine pituitary extract $(50 \mu \mathrm{g} / \mathrm{ml})$, epidermal growth factor $(0.1 \mathrm{ng} / \mathrm{ml})$, penicillin $(100 \mathrm{U} / \mathrm{ml})$, streptomycin $(100 \mu \mathrm{g} / \mathrm{ml})$, and $\mathrm{CaCl}_{2}(0.4 \mathrm{mM})$.

Virus production. Vesicular stomatitis virus glycoprotein (VSV-G)-pseudotyped replicationincompetent HIV particles with enhanced green fluorescent protein (GFP) (Laboratories, Mountain View, CA) in place of the nef gene were generated as previously described [23].

\section{HIV infection and cell viability assay}


Virus was cultured with activated T cells in the presence of AMPs at various

\section{HIV p24 release assay}

HIV-VSV-G was incubated with PBS or AMPs at various concentrations for $30 \mathrm{~min}$ in supplemented RPMI medium. Medium was then diluted 1:20 in assay buffer without detergent and analyzed for the presence of viral core protein p 24 by ELISA. Total p 44 was calculated for each sample using linear regression analysis from p24 standards included on each plate as previously described $[15,28]$. Plates were analyzed by microplate reader (Molecular Devices, Sunnyvale, CA) at $405 \mathrm{~nm}$ absorbance.

\section{LDH Cytotoxicity Assay (Roche)}

The effects of AMPs on viability of the endocervical cell line were measured using a lactate dehydrogenase (LDH) detection kit (Roche Molecular Branchburg, New Jersey) according to manufacturer's directions. The kit measures LDH released into the medium by damaged cells. Briefly, the cells were grown as monolayers adherent to the bottom of 96 -well microtiter plates. Prior to addition of the peptides, spent media was removed and replaced with $200 \mu \mathrm{l}$ fresh media containing the peptide at various dilutions. The cells were incubated with the test agent for 3 hours at $37^{\circ} \mathrm{C}$. After centrifugation of the plates, $100 \mu 1$ of medium was removed from each well for assay and $100 \mu \mathrm{l} /$ well of the $\mathrm{LDH}$ reaction mixture was added. The mixture was incubated for 30 minutes at room temperature protected from light. Absorbance readings at $492 \mathrm{~nm}$ were determined using a microtiter plate reader (Dynex Technologies, Inc., Chantilly, VA). Fresh medium lacking test agent served as the low background control and medium containing 2\% Triton X-100 served as the control for maximal killing and LDH release. 


\section{Results}

Caerin 1 peptides inhibit HIV infection of T cells

Because we had previously shown that several members of the caerin 1 family of amphibian AMPs were effective inhibitors of HIV infection of T cells (caerin 1.1 and caerin 1.9) [25], we examined the activity of 18 additional naturally occurring caerin 1 family peptides or synthetically modified analogs (Table 1). Among these peptides, five (caerin 1.6, caerin 1.7, caerin 1.19 , caerin $1.1 \bmod 7$, and caerin $1.1 \bmod 9$ ) were significantly better inhibitors of HIV infection than our previous best candidate, caerin 1.9 (Fig. 1A). Four other peptides (caerin 1.2, caerin 1.5 , caerin 1.10 , and caerin $1.9 \mathrm{sm}$ ) had nearly identical activity as caerin 1.9 (Fig. 1B). Two other peptides (caerin 1.1 mod 10 and caerin 1.20) had slightly less activity than caerin 1.9 (Fig. 1C), and the remaining seven peptides (caerin 1.3, caerin 1.4, caerin $1.1 \bmod 5$, caerin 1.1 $\bmod 6$, caerin $1.1 \bmod 8$, caerin $1.1 \bmod 11$, and caerin $1.1 \bmod 12)$ had significantly less activity than caerin 1.9 (Figs. S1A and S1B).

\section{Caerin 1 peptides inactivate HIV by disruption of the viral envelope and release of p24 core protein}

We previously showed that caerin 1.9 does not interfere with cellular components required for HIV infection, and it does not interfere with any stage of the HIV life cycle after reverse transcription. It does, however, inhibit viral fusion to target cells by disruption of the viral envelope and release of p 24 core protein [25]. In this assay, p24 can only be detected if the viral envelope is disrupted by the peptide. To determine if the additional caerin 1 peptides also disrupt the viral envelope, the viruses and peptides were incubated in complete RPMI medium for 30 minutes. The medium containing released p 24 was then diluted in assay buffer without detergent and analyzed. Like caerin 1.9, all of the caerin 1 family peptides tested released significant amounts of p24 in a dose-dependent fashion (Fig. 2). Among the peptides tested, some released slightly more $\mathrm{p} 24$ at the lowest concentrations tested $(0.8,1.6,3.12$, and $6.25 \mu \mathrm{M})$ than caerin 1.9 (caerin 1.6, caerin 1.7, caerin 1.19, and caerin $1.1 \bmod 7$ (Fig. 2A). All of the others resulted in the release of p24 in a pattern that was very similar to that of caerin 1.9 over an identical range of concentrations (caerin $1.9 \mathrm{sm}$, caerin $1.1 \bmod 9$, caerin 1.5, caerin $1.1 \bmod 10$, caerin 1.20 , caerin 1.2 , caerin 1.10 , caerin 1.3 , caerin 1.4 , caerin $1.1 \bmod 5$, caerin $1.1 \bmod 6$, caerin $1.1 \bmod 8$, caerin $1.1 \bmod 11$, and caerin $1.1 \bmod 12)($ Figs. 2B, 2C, and 2D). The pattern of p24 release was strongly correlated with inhibition of infection for all peptides tested (Figs. 3 and S2). In Fig. 3, the percent infection and p 24 released by each caerin 1 family member at a concentration of $6.25 \mu \mathrm{M}$ is shown as an individual data point. There was a strong negative correlation between the percent of infection of $\mathrm{T}$ cells by the virus and the release of $\mathrm{p} 24$ by the peptides (Pearson correlation analysis $\mathrm{R}=-0.904, p \leq 0.01$ ). To further illustrate this point, percent infection and $\mathrm{p} 24$ released by treatment with caerin 1.9 , caerin 1.6 , caerin 1.7 , and caerin 1.19 are plotted on the same set of axes in Fig. S2. As the percent infection decreased with increasing concentrations of peptide, the amount of p24 released was increased. Thus, as the ability of the peptide to disrupt the viral envelop improved resulting in the release of more p24, the percent infection decreased.

Caerin 1 family peptides have variable effects on viability of target $T$ cells and vaginal epithelial cells 
Two methods were used to determine viability of target $\mathrm{T}$ cells following exposure to virus and caerin 1 peptides. The first measure of viability was PI exclusion. By PI exclusion, five peptides (caerin 1.10, caerin $1.9 \mathrm{sm}$, caerin $1.1 \mathrm{mod} 9$, caerin 1.2 , and caerin 1.20) showed very low toxicity over a range of concentrations up to about $12.5 \mu \mathrm{M}$ similar to caerin 1.9 (Fig. 4). In contrast, caerin 1.5 , caerin 1.6 , caerin 1.7 , caerin 1.19 , caerin $1.1 \bmod 7$, and caerin 1.1 mod 10 had a narrower range of effectiveness versus viability. Each of these peptides was quite effective in the inhibition of infection and in the release of p24; however, at $12.5 \mu \mathrm{M}$, viability of target cells was reduced (Fig. S3). The other peptides including caerin 1.3, caerin 1.4, caerin 1.1 $\bmod 5$, caerin $1.1 \bmod 6$, caerin $1.1 \bmod 8$, caerin $1.1 \bmod 11$, and caerin $1.1 \bmod 12$ were somewhat less effective inhibitors of infection; and they were non-toxic or only modestly toxic at the concentrations tested (Fig S4).

As a more stringent measure of viability and the capacity to support HIV infection, we determined the ability of target $\mathrm{T}$ cells to proliferate following exposure to caerin 1 peptides. The results were very consistent with those observed for PI exclusion. As shown by proliferation index, viability was excellent over a range of concentrations that inhibit virus infection up to a concentration of about $10 \mu \mathrm{M}$ for caerin 1.10 , caerin $1.9 \mathrm{sm}$, caerin $1.1 \bmod 9$, caerin 1.2 , and caerin 1.20, as well as caerin 1.9 (Fig. 5). Other peptides that were less effective inhibitors of HIV infection (caerin 1.3, caerin 1.4, caerin $1.1 \bmod 6$, caerin $1.1 \bmod 8$, and caerin $1.1 \bmod 11$ ) supported virtually uninterrupted $\mathrm{T}$ cell proliferation up to at least $25 \mu \mathrm{M}$ concentration (Fig. S5).

Although the viability of T cell targets is an important measure of the lack of significant toxicity of the caerin 1 peptides, we also monitored the effects of the peptides on vaginal epithelial cells. For some peptides, the effects on viability of an endocervical cell line were very similar to their effects on $\mathrm{T}$ cells. The peptides with the best viral inactivation profile for $\mathrm{T}$ cells and least toxicity to the vaginal cells were caerin 1.2 , caerin 1.10 , and caerin 1.20 . Caerin 1.5 , caerin 1.6, and caerin 1.7 caused slightly greater loss of viability of the endocervical cells (Fig. 6). Also very effective inhibitors of viral infection of $\mathrm{T}$ cells but more toxic to vaginal cells were caerin $1.9 \mathrm{sm}$, caerin $1.1 \bmod 9$, caerin 1.19 , and caerin $1.1 \bmod 10$. Surprisingly, caerin 1.9 and caerin $1.1 \bmod 7$ were quite toxic to endocervical cells in this assay (Fig. 7). Other peptides that were less effective inhibitors of HIV were also less toxic to endocervical cells (caerin 1.3, caerin 1.4, caerin $1.1 \bmod 6$, and caerin $1.1 \bmod 8$ ) (Fig. S6). Two additional peptides (caerin $1.1 \mathrm{mod}$ 5 and caerin $1.1 \mathrm{mod} 12$ ) were moderately effective inhibitors of HIV infection, but viability of endocervical cells was greatly decreased at the effective concentrations (Fig. S6).

\section{Discussion}

Caerin 1 peptides are effective inhibitors of HIV infection with low toxicity to host cells and endocervical cells

Antimicrobial peptides are produced abundantly in the granular glands of many amphibian species $[1,4,6]$, and they appear to be constitutively released in low quantities to bathe the mucosal surface and inhibit invasion by pathogens [18]. Thus, they have evolved to be effective but relatively non-toxic antimicrobials that attack the fundamental structures of bacterial membranes and enveloped viruses [30]. Previously, we showed that fourteen naturally produced amphibian antimicrobial peptides could inhibit HIV infection of human T cells [25], and two were shown to inhibit transmission of the virus after capture by dendritic cells [25]. Here we show that five additional caerin 1 peptides (caerin 1.2, caerin $1.9 \mathrm{sm}$, caerin 1.10 , caerin 
1.20, and caerin $1.1 \bmod 9$ ) have excellent properties of viral inhibition at low concentrations and low toxicity to T cells (Fig. 4). In this report, we also examined the effects of the caerin 1 peptides on viability of an endocervical call line as a proxy for possible toxicity to vaginal epithelial cells. A number of the peptides effectively inhibited virus infection of $\mathrm{T}$ cells at concentrations between 6-12 $\mu \mathrm{M}$ and did not impair endocervical cell viability at these concentrations (caerin 1.2, caerin 1.5, caerin 1.6, caerin 1.7, caerin 1.10, and caerin 1.20) (Fig. 6). Thus, they would appear to be safe for use as microbicides at this range of concentrations.

The mechanism of action of the entire family of caerin 1 peptides tested is the disruption of the viral envelope and release p 24 core protein. The more effective they were at disruption of the viral envelope, the better they were at inhibiting infection (Fig. 3 and S2). Thus, some peptides that were highly effective in the inhibition of viral infection, such as caerin 1.5 , caerin 1.6 and caerin 1.7, caerin $1.9 \mathrm{sm}$, caerin $1.1 \mathrm{mod} 7$, and caerin $1.1 \mathrm{mod}$; were able to disrupt the HIV envelope and release elevated amounts of p24 but were also more toxic to T cells or endocervical cells.

\section{Potential benefits and drawbacks to the use of caerin peptides as anti-HIV microbicides}

In addition to the capacity of the caerin peptides to directly inhibit HIV by disruption of the viral envelope, it is quite likely that the peptides may also have other immunomodulatory activities distinct from their antiviral activities. A number of recent papers have demonstrated the capacity of amphibian AMPs to affect lymphocyte proliferation and cytokine production $[9$, $17,19$, reviewed in 7$]$. Several peptides, including caerin 1.8 , have the capacity to interact with calmodulin, a critical signaling molecule in T lymphocyte activation [5]. The immunomodulatory activities include both potentially enhancing and suppressive activities [7].

As suggested by others [8], cationic peptides with membrane-disrupting potential may have relatively poor therapeutic indices (the ratio of the concentration at which host cell viability is reduced by $50 \%$ to the concentration at which viral infection is reduced by $50 \%$ ). We estimate that the therapeutic index (TI) of the most effective caerin 1 peptides would be 20 or less as estimated in these in vitro conditions. These results are similar to estimates of TIs reported previously following identification of additional anti-HIV inhibitory peptides using the AMP database and AMPs derived from human and bovine cathelicidins [26, 27].

The vaginal microenvironment would also likely be rich in proteases which could degrade the peptides over time. This could be potentially beneficial because the peptide activity is self-limited and would not persist long-term to induce possible cytotoxic effects. Amphibian skin has some correlates with other mucosal surfaces. Recent studies showed that antimicrobial peptides secreted in the mucus of the leopard frog persisted for approximately 2 hours, but the amounts were greatly decreased by 30 to 60 minutes after secretion due to proteolytic cleavage [17]. One possible method to retain higher concentrations of active peptides would be the substitution of some D-amino acids during synthesis to produce D-peptides with possible greater resistance to naturally occurring proteases [24]. Another approach would be the recombinant expression in commensal lactobacilli which inhabit the vagina. These bacteria produce their own defensive peptides and lactic acid to inhibit other potential pathogenic microbes. If they were engineered to secrete additional antimicrobial peptides, they might generate an environment more hostile to HIV. Studies of the use of protective lactobacilli are moving forward and show considerable promise [10,21].

Cationic peptides have the potential to be chemotactic for immune cells and induce production of chemokines by the activated populations [3,17, 19, 20, reviewed in 7]. This could 
be beneficial to enhance possible anti-HIV antibody responses. Alternatively, it could have unwanted side effects. A recent study of the enhancement of HIV infection in the presence of co-infection with herpes simplex virus type 2 (HSV-2) showed that HSV-2 infection of epithelial cells induced expression of the cationic peptide LL-37, and LL-37 likely upregulated expression of CD4 and the coreceptor for HIV CCR5 on Langerhans cells possibly increasing their susceptibility to HIV infection [14]. However, the situation is more complex because LL37 itself can inhibit HIV $[2,26]$. Thus, the interplay of normal vaginal cells with the vaginal microbiome and introduced viruses is very complex, and additional research is needed to determine whether and to what extent introduction of antimicrobial peptides such as caerin 1 peptides would be beneficial.

\section{Acknowledgements}

This work was supported by AMFAR (American Foundation for AIDS Research) grant (to DU and LAR-S) and NSF grant IOS-1121758 (to LAR-S). We thank Paavan Kotini for assistance with the HIV p24 release assay.

\section{References}

1. Apponyi MA, Pukala TL, Brinkworth CS, Maselli VM, Bowie JH, Tyler MJ, Booker GW, Wallace JC, Carver JA, Separovic F, Doyle J, Llewellyn LE. 2004. Host defense peptides of Australian anurans: structure, mechanism of action and evolutionary significance. Peptides 25:1035-1054.

2. Bergman P, Walter-Jallow L, Broliden K, Agerberth B, Söderlund J. 2007. The antimicrobial peptide LL-37 inhibits HIV-1 replication. Curr HIV Res. 5:410-415.

3. Bommineni YR, Pham GH, Sunkara LT, Achanta M, Zhang G. 2014. Immune regulatory activities of fowlicidin-1, a cathelicidin host defense peptide. Mol. Immunol. 59:55-63.

4. Bowie JH, Separovic F, Tyler MJ. 2012. Host-defense peptides of Australian anurans. Part 2. Structure activity, mechanism of action, and evolutionary significance. Peptides 37:174-188.

5. Calabrese AN, Bowie JH, Pukala TL. 2015. Structural analysis of calmodulain binding by nNOS inhibitory amphibian peptides. Biochemistry 54:567-576.

6. Conlon JM. 2011. Structural diversity and species distribution of host-defense peptides in the frog skin secretions. Cell. Mol. Life Sci 68:2303-2315.

7. Conlon JM, Mechkarska M, Lukic ML, Flatt PR. 2014. Potential therapeutic applications of multifunctional host-defense peptides from frog skin as anti-cancer, and-vial, immunomodulatory, and anti-diabetic agents. Peptides 57:67-77.

8. Eade C R, Wood MP, Cole AM. 2012. Mechanisms and modifications of naturally occurring host defense peptides for anti-HIV microbicide development. Curr. HIV Res.10:61-72.

9. Jackway RJ, Pukala TL, Maselli VM, Musgrave IF, Bowie JH, Liu Y, Surinya-Johnson KH, Donnellan SC, Doyle JR, Llewellyn LE, Tyler MJ. 2008. Disulfide-containing peptides from the glandular skin secretions of forglets of the genus Crinia: structure, activity and evolutionary trends. Regul. Pept. 151:80-87

10. Lagenaur LA, Sanders-Beer BE, Brichacek B, Pal R, Liu X Liu Y, Venzon D, Lee PP, Hamer DH. 2011. Prevention of vaginal SHIV transmission in macaques by a live recombinant Lactobacillus. Mucosal. Immunol. 4:648-657. 
11. Lorin C, Saidi H, Belaid A, Zairi A, Baleux F, Hocini H, Bélec L, Hani K, Tangy F. 2005. The antimicrobial peptide dermaseptin S4 inhibits HIV-1 infectivity in vitro.

12. McDonald D, Wu L, Bohks SM, KewalRamani VN, Unutmaz D, Hope TJ. 2003. Recruitment of HIV and its receptors to dendritic cell-T cell junctions. Science 300:12951297.

13. Nicolas P, Mor A. 1995. Peptides as weapons against microorganism in the chemical defense system of vertebrates. Annum Rev. Microbiol 49:277-304.

14. Ogawa Y, Kawamura T, Matsuzawa T, Aoki R, Gee P, Yamashita A, Moriishi K, Yamasaki K, Koyanagi Y, Blauvelt A, Shimada S. 2013. Antimicrobial peptide LL37 produced by HSV-2-infected keratinocytes enhances HIV infection of Langerhans cells. Cell Host Microbe. 13:77-86.

15. Oswald-Richter K, Grill SM, Leelawong M, Unutmaz D. 2004. HIV infection of primary human $\mathrm{T}$ cells is determined by tunable thresholds of T cell activation. Eur. J. Immunol 34:1705-1714.

16. Oswald-Richter K, Grill SM, Shariat N, Leelawong M, Sundrud MS, Haas DW, Unutmaz D. 2004. HIV infection of naturally occurring and genetically reprogrammed human regulatory T-cells. PLoS Biol. 2:E198.

17. Pantic JM, Mechkarska M, Lukic ML, Conlon JM. 2014. Effects of tigerinin peptides on cytokine production by mouse peritoneal macrophages and spleen cells and by human peripheral blood mononuclear cells. Biochimie 101: 83-92

18. Pask JD, Woodhams DC, Rollins-Smith LA. 2012. The ebb and flow of antimicrobial skin peptides defends northern leopard frogs (Rana pipiens) against chytridiomycosis. Global Change Biol. 18:1231-1238.

19. Popovic S, Urbán E, Lukic M, Conlon JM. 2012. Peptides with antimicrobial and antiinflammatory activities that have therapeutic potential for treatment of acne vulgaris. Peptides 34:275-282.

20. Pundir P, Catalli A, Leggiadro C, Douglas SE, Kulka M. 2014. Pleurocidin, a novel antimicrobial peptide, induces human mast cell activation through the FPRL1 receptor. Mucosal Immunol. 7:177-187.

21. Pusch O, Kalyanaraman R, Tucker LD, Wells JM, Ramratnam B, Boden D. 2006. An anti-HIV microbicide engineered in commensal bacteria: secretion of HIV-1 fusion inhibitors by lactobacilli. AIDS. 20:1917-1922.

22. UNAIDS. Global Report: UNAIDS Report on the Global AIDS Epidemic [home page for global fact sheet cited 2015 May 7] http://www.unaids.org/en/resources/campaigns/globalreport2013/globalreport

23. Unutmaz D, KewalRamani VN, Marmon S, Littman DR. 1999. Cytokine signals are sufficient for HIV-1 infection of resting human T lymphocytes. J. Exp. Med. 189:17351746.

24. Van Regenmortel MH, Muller S. 1998. D-peptides as immunogens and diagnostic reagents. Curr. Opin. Biotechnol. 9:377-382.

25. VanCompernolle S E, Taylor R J, Oswald-Richter K, Jiang J, Youree B E, Bowie J H, Tyler MJ, Conlon J M, Wade D, Aiken C, Dermody T S, KewalRamani V N, RollinsSmith L A, Unutmaz D. 2005. Antimicrobial peptides from amphibian skin potently inhibit human immunodeficiency virus infection and transfer of virus from dendritic cells to T cells. J. Virol. 79:11598-11606. 
364 26. Wang G, Watson K, Buckheit R Jr. 2008. Anti-human immunodeficiency virus type 1 365 (HIV-1) activities of antimicrobial peptides derived from human and bovine

27. Wang G, Watson KM, Peterkofsky A, Buckheti RW Jr. 2010. Identification of novel human immunodeficiency virus type 1-inhibitory peptides based on the antimicrobial

28. Wehrly K, Chesebro B. 1997. p24 antigen capture assay for quantification of human immunodeficiency virus using readily available inexpensive reagents. Methods 12:288293.

29. Zairi A, Tangy F, Bouassidia K, Hani K. 2009. Dermaseptins and magainins: Antimicrobial peptides from frogs'skin-New sources for promising spermicides microbicides-A mini review. J. Biomed. Biotechnol. 2009:452567.

30. Zasloff M. 2002. Antimicrobial peptides of multicellular organisms. Nature 415:389-395. 
379 TABLE 1. Caerin 1 family peptides and synthetic analogs

\begin{tabular}{|c|c|c|c|}
\hline Peptide & Species of origin & Sequence & Mass \\
\hline Caerin $1.1^{*}$ & Litoria caerulea & GLLSVLGSVAKHVLPHVVPVIAEHL-NH ${ }_{2}$ & 2582 \\
\hline Caerin $1.1 \bmod 5$ & synthetic & GLLSVLGSVAKHVLAHVVAVIAEEHL-NH $_{2}$ & 2530 \\
\hline Caerin $1.1 \bmod 6$ & synthetic & GLLSVLGSVAKHVLPHVVPVIAEHA-NH ${ }_{2}$ & 2540 \\
\hline Caerin $1.1 \bmod 7$ & synthetic & GLLSVLGSVAKHVLPHVVPVIAAAL-NH $_{2}$ & 2458 \\
\hline Caerin $1.1 \bmod 8$ & synthetic & GLLSVLGSVAKHVLPHVVPVIAEEL-NH ${ }_{2}$ & 2574 \\
\hline Caerin $1.1 \bmod 9$ & synthetic & GLLSVLGSVAKHVLPHVVPVIAKLH-NH $_{2}$ & 2581 \\
\hline Caerin $1.1 \bmod 10$ & synthetic & GLLKVLGSVAKKVLPKVVPVIAEKL-NH $_{2}$ & 2596 \\
\hline Caerin $1.1 \bmod 11$ & synthetic & GLLKSLSSVAKKVLPKVVPVIAEKL-NH ${ }_{2}$ & 2614 \\
\hline Caerin $1.1 \bmod 12$ & synthetic & GLLKKLKKVAKKVLPKVVPVIAEKL-NH ${ }_{2}$ & 2737 \\
\hline Caerin 1.2 & L. caerulea & GLLGVLGSVAKHVLPHVVPVIAEHL-NH $_{2}$ & 2552 \\
\hline Caerin 1.3 & L. caerulea & GLLSVLGSVAQHVLPHVVPVIAEHL-NH $_{2}$ & 2582 \\
\hline Caerin 1.4 & L. caerulea, L. gilleni & GLLSSLSSVAKHVLPHVVPVIAEHL-NH $_{2}$ & 2600 \\
\hline Caerin 1.5 & L. caerulea & GLLSVLGSVVKHVIPHVVPVIAEHL-NH $_{2}$ & 2610 \\
\hline Caerin 1.6 & $\begin{array}{l}\text { L. splendida, } L \text {. } \\
\text { xanthomera }\end{array}$ & GLFSVLGAVAKHVLPHVVPVIAEKL-NH $_{2}$ & 2594 \\
\hline Caerin 1.7 & $\begin{array}{l}\text { L. chloris, L. } \\
\text { xanthomera }\end{array}$ & GLFKVLGSVAKHLLPHVAPVIAEKL-NH ${ }_{2}$ & 2637 \\
\hline Caerin 1.9 & L. chloris & GLFGVLGSIAKHVLPHVVPVIAEKL-NH $_{2}$ & 2591 \\
\hline Caerin $1.9 \mathrm{sm}$ & synthetic & GLFGVLGSIAKHLLPHVVPVIAEKL-NH $_{2}$ & 2605 \\
\hline Caerin 1.10 & L. splendida & GLLSVLGSVAKHVLPHVVPVIAEKL-NH $_{2}$ & 2573 \\
\hline Caerin 1.19 & L. gracilenta & GLFKVLGSVAKHLLPHVAPIIAEKL-NH $_{2}$ & 2649 \\
\hline Caerin 1.20 & $\begin{array}{l}\text { L. caerulea/L. } \\
\text { splendida }\end{array}$ & GLFGILGSVAKHVLPHVIPVVAEHL-NH $_{2}$ & 2600 \\
\hline
\end{tabular}

Amino acid modifications to the caerin 1.1 peptides are shown in bold type with gray shading. *Caerin 1.1 was tested previously (6) and not retested in these studies. Its sequence is shown 382 here for comparison with the synthetically modified caerin 1.1 peptides. 
Figure 1. Caerin 1 peptides inhibit HIV infection of T cells. (A -C) The capacity to inhibit HIV infection by the caerin 1 family peptides shown was compared with that of caerin 1.9. *, For panel A, inhibition at $1.6 \mu \mathrm{M}, 3.25 \mu \mathrm{M}$, and $6.5 \mu \mathrm{M}$ was significantly greater than that of caerin

1.9 at the same concentration by two-tailed Student's t-tests, $p \leq 0.02$. For panel B, inhibition at each data point was not different from that of caerin 1.9 at all concentrations. For panel $\mathrm{C},{ }^{*}$, Inhibition by caerin 1.20 at $3.25 \mu \mathrm{M}, 6.5 \mu \mathrm{M}$, and $12.5 \mu \mathrm{M}$ was significantly less than that of caerin 1.9 at the same concentrations; \#, Inhibition by caerin $1.1 \bmod 10$ was significantly less than that of caerin 1.9 at $12.5 \mu \mathrm{M}$ by two-tailed Student's t-test $\mathrm{p} \leq 0.02$. Data shown are for three replicate samples for each parameter tested in one representative experiment of two independent experiments. Error bars indicate standard errors.

Figure 2. Caerin 1 peptides disrupt the HIV viral envelope and release p24 core protein. (A-D) The capacity to disrupt the viral envelope of the caerin 1 family peptides shown in each panel was compared with that of caerin 1.9. For panel A*, Significantly greater release of p24 than caerin 1.9 at the same concentrations by two-tailed Student's t-tests, $p \leq 0.0552$. For panel B-D, release of p24 was not different or greater than that of caerin 1.9. Data shown are for three replicate samples for each parameter tested in one experiment. Error bars indicate standard errors.

Figure 3. Release of p 24 is negatively correlated with viral infectivity. The percent infection (yaxis) observed and the 24 released ( $\mathrm{x}$-axis) by each of the caerin 1 family peptides listed in Table 1(except caerin 1.1) at a concentration of $6.25 \mu \mathrm{M}$ is plotted. The best-fit linear regression revealed a negative slope of 294.5. (Pearson correlation analysis $\mathrm{R}=-0.904, p,<0.01$ ).

Figure 4. The caerin 1 peptides shown inhibit viral infection of $\mathrm{T}$ cells without impairing viability over a broad concentration range. The capacity of caerin 1 family peptides to inhibit HIV infection of T cells was determined as described in methods. Their effects on T cell viability at the same concentrations in the same experiment was determined by propidium iodide (PI) exclusion. Data shown are for three replicate samples for each parameter tested in one representative experiment of two independent experiments. Error bars indicate standard errors.

Figure 5. Some caerin 1 peptides inhibit viral infection of $\mathrm{T}$ cells over a broad concentration range without impairing the ability of the $\mathrm{T}$ cells to proliferate. All peptides were incubated at the concentrations shown with $3 \times 10^{4}$ infectious units of HIV-VSV-G-GFP for 15 minutes. Viral-peptide solutions were diluted in complete RPMI medium with $3 \times 10^{4}$ activated human $\mathrm{CD}^{+} \mathrm{T}$ cells to achieve an MOI of 1 and incubated at $37^{\circ} \mathrm{C}$ for 3 days. Cells were harvested and analyzed for GFP expression by flow cytometry as an indicator of virus infection. To determine effects of peptides on proliferation, peptides at the concentrations shown were incubated for 5 minutes with $5 \times 10^{4} \mathrm{CFSE}$-labeled primary $\mathrm{CD} 4^{+} \mathrm{T}$ cells. Cells were then diluted with complete RPMI medium and activated by T-cell receptor engagement as previously described [16]. The activated cells were cultured for 4 days, and CFSE fluorescence intensity was measured by flow cytometry. The proliferation index was calculated as the sum of the total cells in all generations divided by the estimated number of original parent cells. Data shown are for three replicate samples for each parameter tested in one experiment. Error bars indicate standard errors. 
$430 \quad$ Figure 6. The caerin peptides shown inhibit viral infection of $\mathrm{T}$ cells over a broad concentration 431 range without impairing the viability of endocervical cells. The capacity of caerin 1 peptides to 432 inhibit HIV infection of T cells was determined as described in methods. The effects of each 433 peptide on viability of the endocervical cells was determined by LDH released into the medium 434 by damaged cells as described in methods. Data shown are for five replicate samples at each 435 parameter tested. Error bars indicate standard errors. Each peptide was tested once or twice.

Figure 7. The caerin 1 peptides shown inhibit viral infection of $\mathrm{T}$ cells effectively, but they are somewhat more toxic to endocervical cells than other members of the caerin 1 family. The capacity of caerin 1 family peptides to inhibit HIV infection of T cells was determined as described in methods. The effects of each peptide on viability of the endocervical cells was determined by LDH released into the medium by damaged cells as described in materials and methods. Data shown are for five replicate samples at each parameter tested. Error bars indicate standard errors. Each peptide was tested once or twice. 


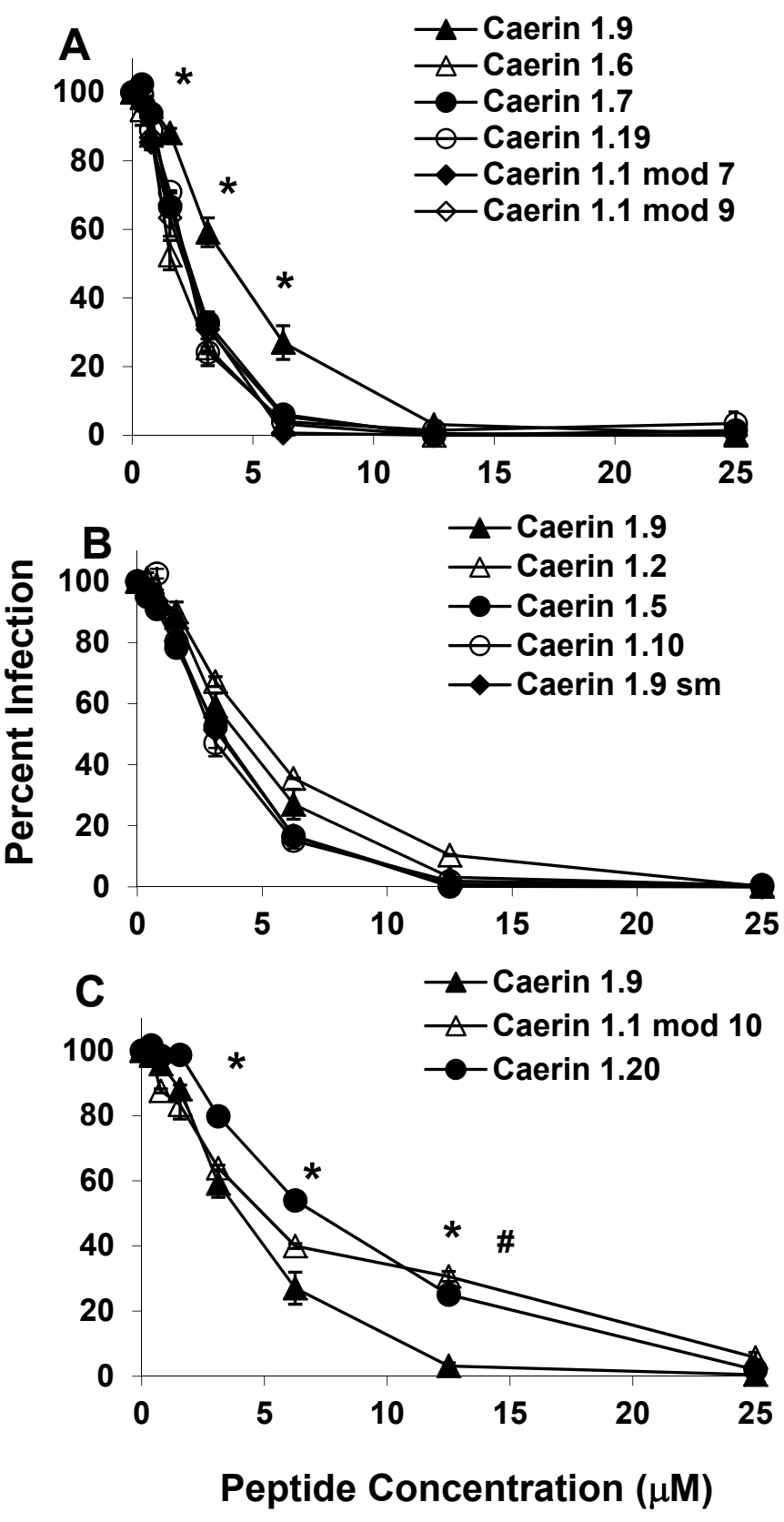

Figure 1. 

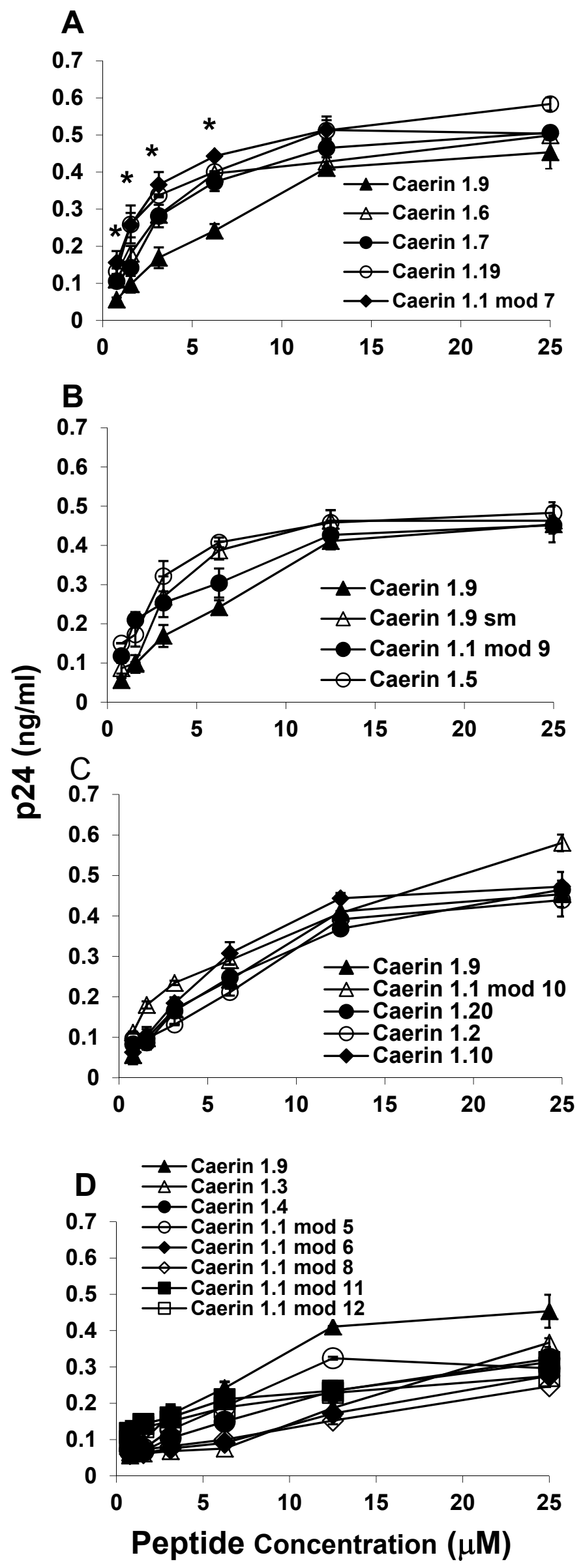

Figure 2. 


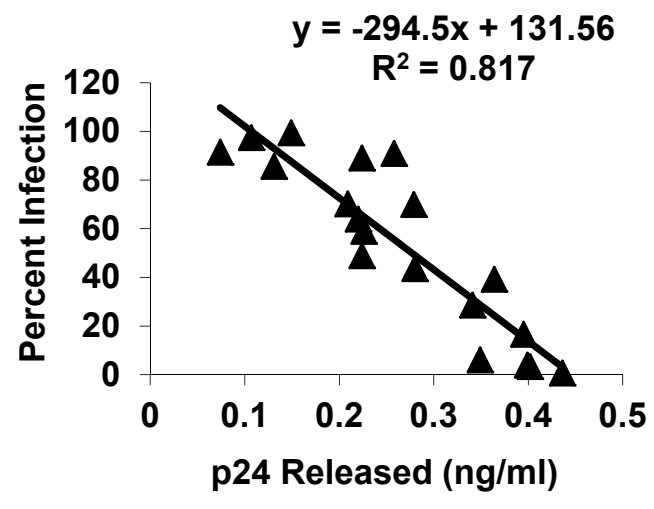

Figure 3. 


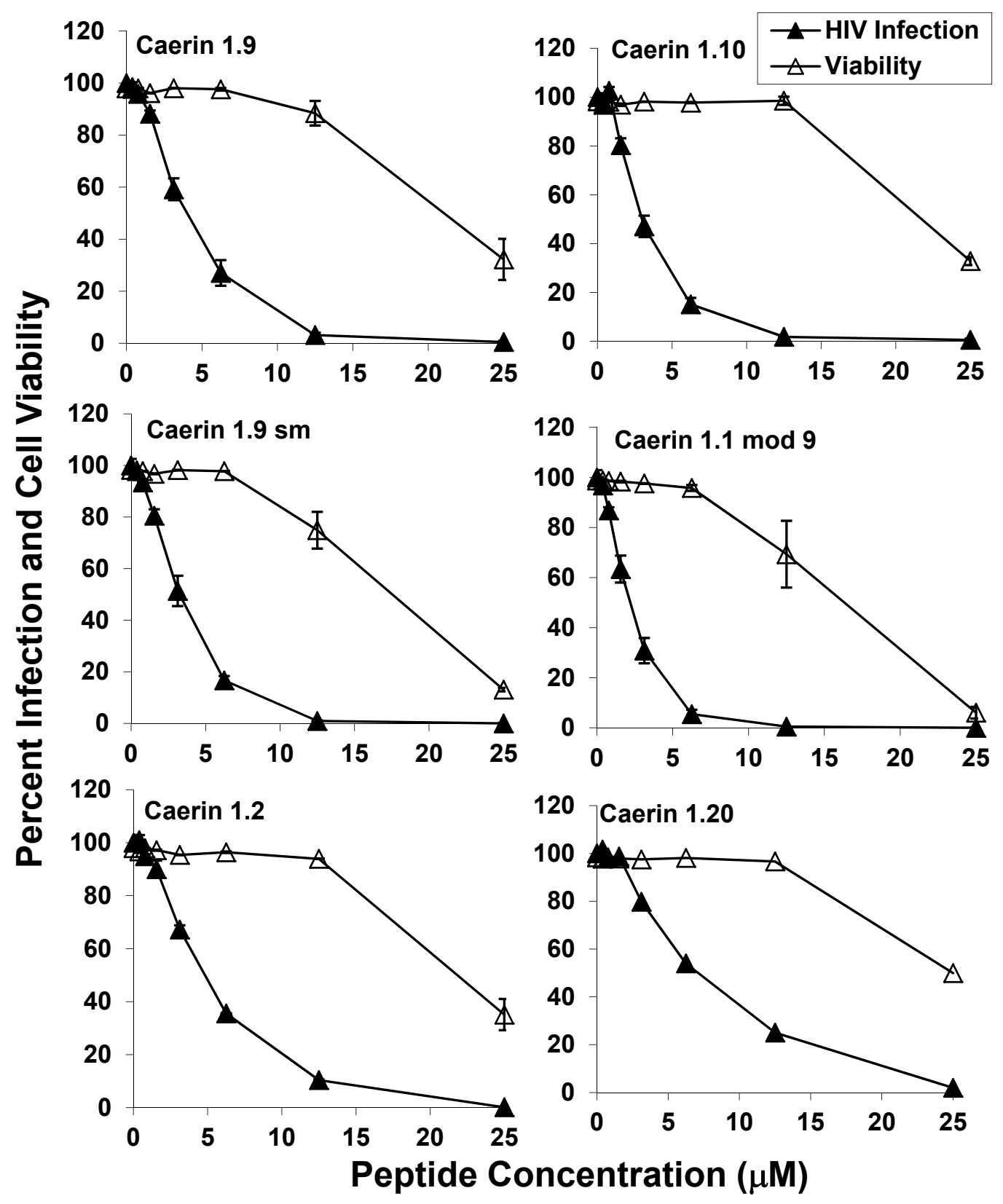

Figure 4. 


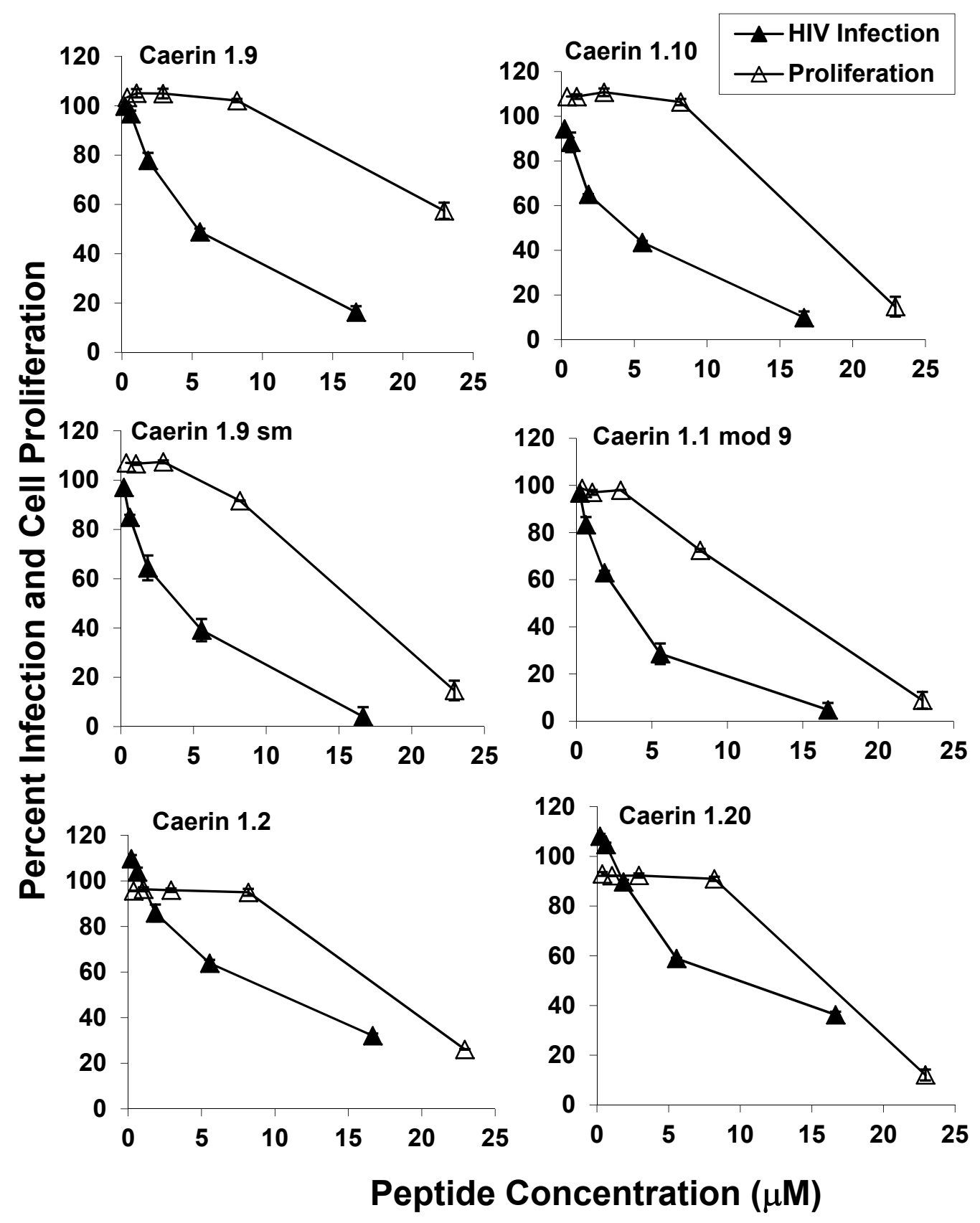

Figure 5. 


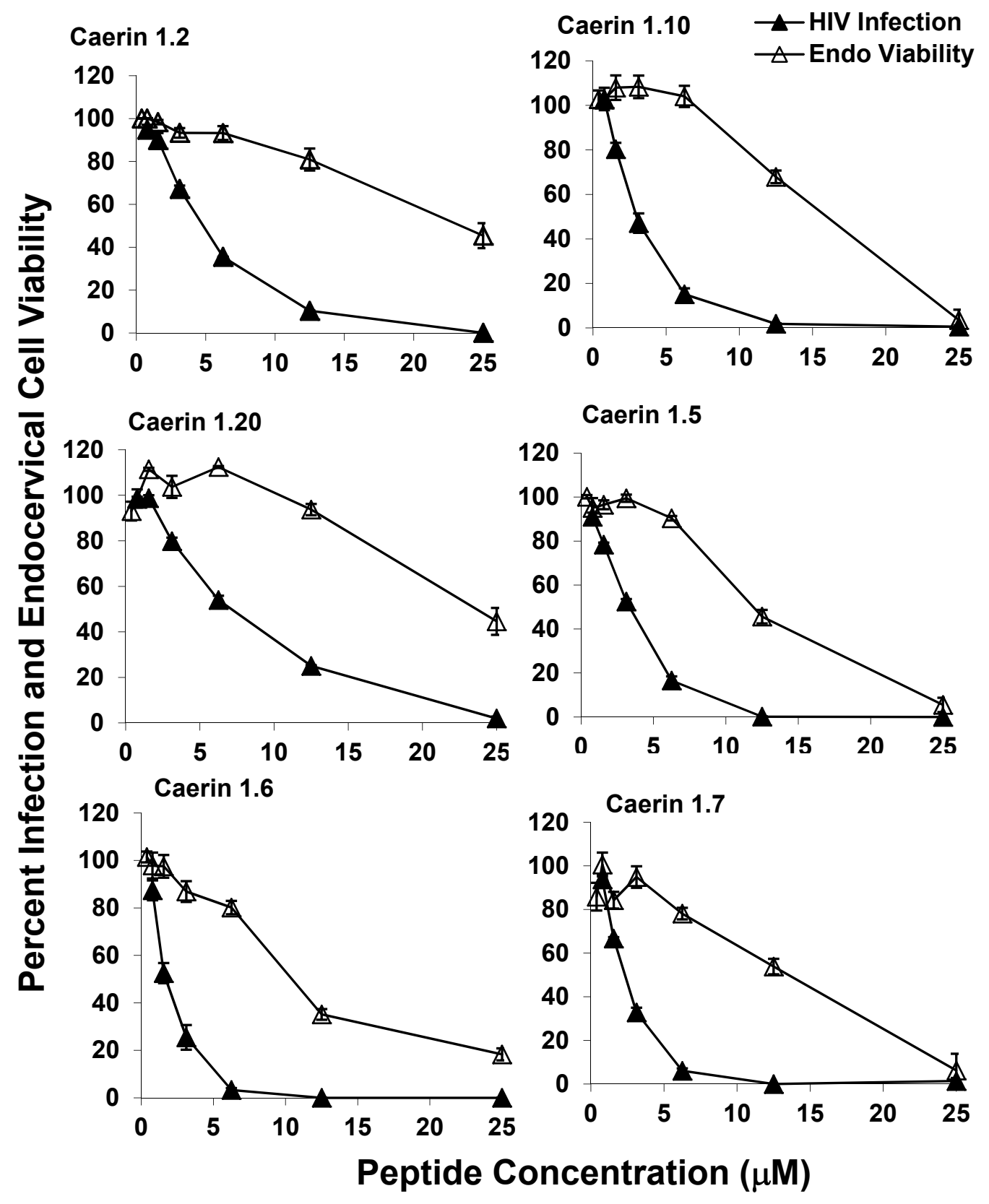

Figure 6. 


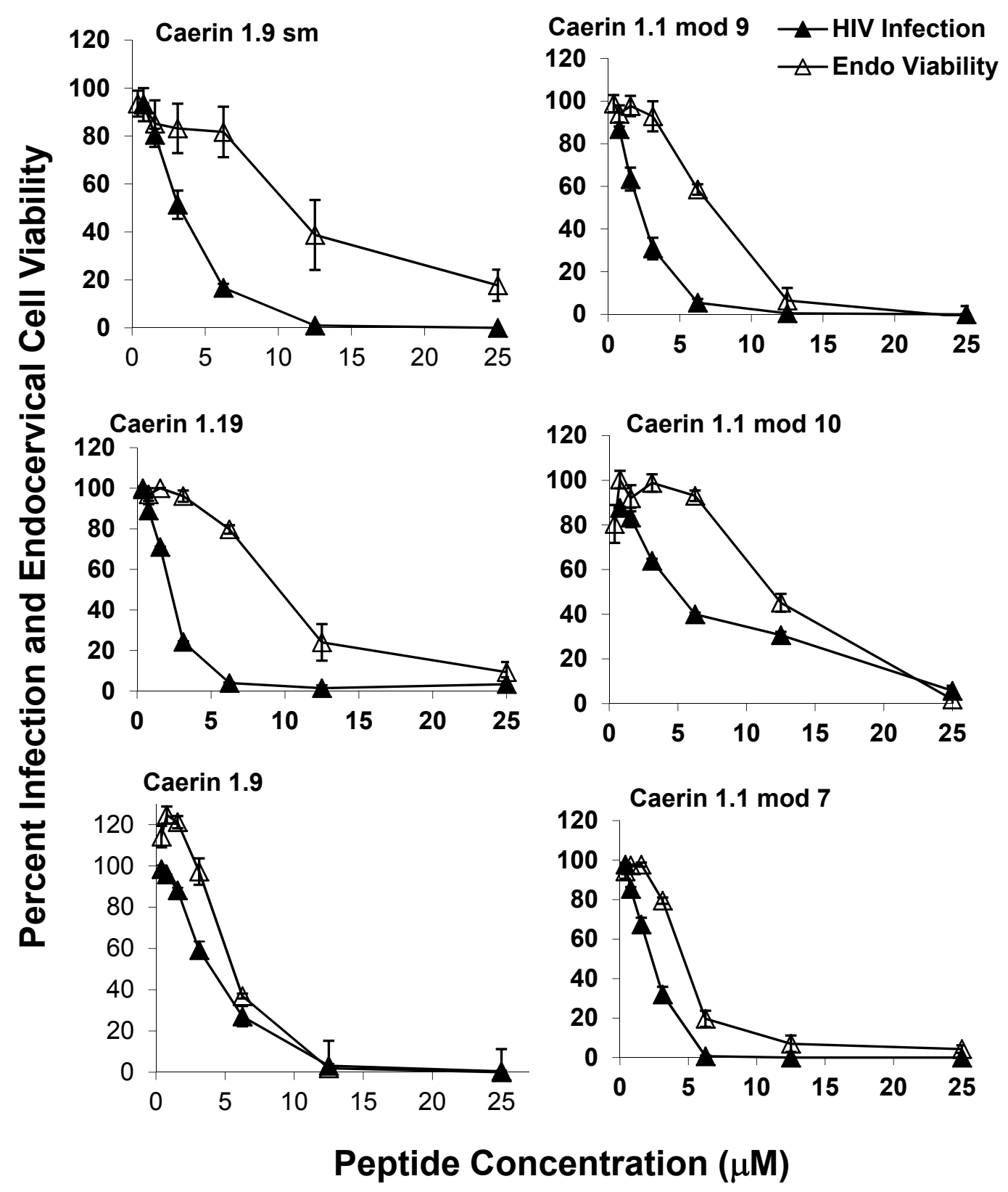

Figure 7. 